\title{
The Role of Behavioral Health in Optimizing Care for Complex Patients in the Primary Care Setting
}

\author{
Martha C. Ward, MD ${ }^{1,2}$, Benjamin F. Miller, PsyD ${ }^{5}$, Vincent C. Marconi, MD ${ }^{3,4}$, Nadine J. Kaslow, PhD , \\ and Eugene W. Farber, $P h D^{1}$
}

'Department of Psychiatry and Behavioral Sciences, Emory University, Atlanta, GA, USA; ${ }^{2}$ Department of Medicine, Division of General Medicine, Emory University, Atlanta, GA, USA; ${ }^{3}$ Department of Medicine, Division of Infectious Diseases, Emory University, Atlanta, GA, USA; ${ }^{4}$ Department of Global Health, Emory University Rollins School of Public Health, Atlanta, GA, USA; ${ }^{5}$ Department of Family Medicine, University of Colorado, Denver, CO, USA.

KEY WORDS: behavioral health; primary care; integrated care; mental health; patient outcomes; provider satisfaction.

J Gen Intern Med 31(3):265-7

DOI: $10.1007 /$ s11606-015-3499-8

(c) Society of General Internal Medicine 2015

$\mathrm{N}$ ever before has so much been asked of primary care. Primary care providers are increasingly encountering heavy workloads, serving a large volume of patients in shorter visit times. At the same time, there is a growing emphasis on healthcare delivery models that meet the "triple aim" (patient satisfaction, improved health outcomes, and lower cost). In the context of these demands, patient complexity in the primary care setting is high, with roughly one in four primary care patients presenting with multi-morbidity ${ }^{1}$ and up to half meeting criteria for a psychiatric disorder. ${ }^{2}$ As such, emerging primary care models must address the significant impact of behavioral health complications on both the complexity and intensity of care. Integrating behavioral health providers (e.g., psychiatrists, psychologists, social workers) into primary care teams can enhance provider responsiveness to patients with complex clinical presentations and improve patient/family outcomes and satisfaction. This approach may also lower stress and increase professional quality of life for primary care physicians, associated specialty care providers, physician's assistants, nurse practitioners, nurses, and other members of the healthcare team.

A key role for behavioral health providers in the primary care setting is to identify and treat psychiatric conditions. Because primary care providers typically have little training in assessing and managing psychiatric conditions, they understandably may experience discomfort in working with some patients with psychiatric comorbidities. In addition to the challenges associated with treatment of psychiatric disorders, unidentified or untreated mental illness can impact overall care, and may be associated with multiple visits for medically unexplained, yet debilitating, somatic symptoms. Such visits can be frustrating for both patients and their care teams, and

Published online August 26, 2015 provider-rated "difficult" encounters are more likely to occur both with patients who have mental health diagnoses and with patients describing multiple somatic complaints. ${ }^{3}$ Behavioral health providers can support their primary care colleagues in managing the behavioral complications of mental health conditions to optimize provider- patient relationships, patient and family engagement in care, and treatment outcomes. They can offer accessible and prompt follow-up treatment when psychiatric conditions are identified, either within the primary care setting itself or via facilitated referral to specialty behavioral health treatment resources outside the primary care practice. They can also provide telephone sessions or utilize telemedicine for patients with limited transportation access to clinic offices.

Behavioral health providers can also support the efforts of the primary care team to facilitate health-enhancing behaviors, address behaviors associated with health risk, and improve pain management and pain-related outcomes by focusing on non-pharmacologic coping strategies. Primary care providers spend a significant portion of their clinical time managing chronic medical conditions, largely through pharmacotherapy. Yet medication management will not optimally alter the course of these diseases if patients engage in adverse health behaviors that contribute to illness progression, or if they are medication non-adherent. Behavioral health providers offer first-line evidence-based interventions for behavioral change, including motivational interviewing and cognitive and behavioral interventions that facilitate patient-directed lifestyle changes. These interventions can increase patient self-management, reduce the frequency and length of clinic visits, and work in synergy with other treatment strategies to improve patient outcomes and support the self-efficacy of primary care providers.

Moreover, behavioral health providers can address psychosocial factors that may influence patient self-care, health behaviors, and participation in care. Complex social challenges can affect the ability of patients to pay for their prescriptions and access safe transportation, creating barriers to adherence to medical recommendations. Family stresses such as violence and loss can increase medical problems and associated 
behavioral health difficulties. Primary care providers often find that they lack the requisite expertise or time to address these psychosocial challenges. Coordinating with their social services colleagues, behavioral health providers can offer supportive interventions to address the emotional impact of psychosocial challenges, help the care team formulate biopsychosocial treatment strategies that consider psychological functioning, and coordinate referrals to appropriate resources.

Given their expertise in understanding interpersonal transactional processes, behavioral health providers also are well positioned to strengthen the individual and team functioning of members of a primary care team. Though challenging patient-provider encounters have historically been viewed as driven primarily by patient factors, there is increasing understanding of the role of reciprocal processes in the patient-provider dyad. ${ }^{3}$ Behavioral health providers can offer consultation with primary care providers to identify patient care strategies that optimize communication and facilitate patient- and familycentered interactions. This may include a focus on culture-specific factors that influence patient health beliefs, behavior, and relationships with healthcare providers. Behavioral health providers also can support the team in troubleshooting systemic factors that may compromise effective patient-provider relationships (such as different perspectives about treatment plans), preventing patient-provider alliance breaches, and resolving such breaches when they do occur. Patient-provider alliance breaches can be stressful for patients and providers alike, and have the potential to negatively impact patient care and health outcomes, and result in medical provider burnout. Often, patient-provider alliance breaches are caused by provider compassion fatigue and frustration. Behavioral health providers can help manage such reactions by serving as a consultative resource in team meetings where providers can safely explore challenging experiences related to the healthcare environment. Behavioral health providers can also facilitate debriefings for individual providers and for the healthcare team when there is a loss of life or another adverse outcome. Additionally, behavioral health providers can serve as a resource for the primary care team in facilitating the delivery of emotionally painful news to patients and their family members, or assist in meetings with patients and their support systems when complex medical decisions must be made.

Incorporating behavioral health providers into primary care teams yields exciting opportunities for interdisciplinary cross-training. Behavioral health providers may share their knowledge with primary care colleagues regarding early detection, diagnosis, and basic treatment of mental health concerns. They can illustrate applications of motivational interviewing and mindfulness skills in the context of medical encounters with patients. This specialty expertise may be disseminated formally, through didactics and multidisciplinary care conferences, or via informal "curbside" consultations and observations of behavioral health intervention practices. These activities can facilitate partnership between behavioral health and primary care practitioners in enacting patient activation and self-management of chronic conditions, and ultimately enhance health-promoting partnerships between patients and healthcare teams.

While there is evidence from the Veterans Health Administration model and elsewhere to suggest that behavioral health integration into primary care settings can improve patient access to care and enhance patient and provider satisfaction, ${ }^{4,5}$ the empirical literature in this area is limited, and more research is needed to determine the effectiveness of this integrated care strategy. In addition, there are challenges with regard to financial sustainability of these care delivery models.

As payment systems move away from a fee-for-service (FFS) framework, primary care models that integrate behavioral health have the potential to increase the value and quality of care. This potential is constrained, however, by limitations of current and new payment models. For instance, many state policies limit billing for medical and behavioral health services on the same day, and FFS reimbursement provides incentives for volume over value, with little consideration for outcomes. FFS often dictates what a provider can do for a patient, and may limit the role of behavioral health providers in primary care settings. Though modified FFS plans include health and behavior assessment codes (which allow billing for a variety of mental health services on the same day of billing by other providers), they still focus on encounter volume rather than quality, and do not fully support team-based care. Bundled payments (which reimburse for a discrete course of treatment) encourage greater team collaboration, but often have FFS underpinnings. In such cases, behavioral health services may not be included within the bundle, or other FFS elements still encourage individual providers to only take fiscal responsibility for themselves rather than for the healthcare team. Global payment structures are perhaps the payment reform model that best supports integration of behavioral health providers into the primary care team. The global budget allows the practice to create the team that will deliver whatever services are most appropriate for the population, and may be combined with pay-forperformance incentives to reward improved patient outcomes. ${ }^{6}$ Even under these models, however, the returns on investing in behavioral health integration are still in need of additional study.

While necessary, payment reform is only one piece that helps lead delivery system transformation to better integrate behavioral health with primary care. Primary care practices 
will need to transform their physical layouts and electronic medical records to accommodate real-time interdisciplinary care provision and information sharing, as well as reconfigure their quality improvement infrastructure. Both primary care and behavioral health providers will need to change their approach to practice. Pursuing changes in educational curricula, including training in team-based and consultative care, will be essential to ensuring the cultivation of a behavioral health workforce that can be integrated within the primary care setting. Such a framework should acknowledge the inseparability of physical and behavioral health services for improving the health of our patients and enhancing professional satisfaction for our healthcare providers.

Acknowledgments: The authors would like to thank the 2014 American Psychological Association Presidential Task Force on Psychology in Patient-Centered Medical Homes for their support and inspiration in the preparation of this article.

Conflict of Interest: The authors have no conflicts of interest.
Corresponding Author: Martha C. Ward, MD; Department of Psychiatry and Behavioral SciencesEmory University, 49 Jesse Hill Jr. Dr. SE, Suite 231, Atlanta, GA 30303, USA (e-mail: mcraig@emory.edu).

\section{REFERENCES}

1. Clark NM. The multiple challenges of multiple morbidities. Health Educ Behav. 2011;38(3):219-21.

2. Toft T, Fink P, Oernboel E, Christensen K, Frostholm L, Olesen F. Mental disorders in primary care: prevalence and co-morbidity among disorders. results from the functional illness in primary care (FIP) study. Psychol Med. 2005;35(8): 1175-84

3. An PG, Rabatin JS, Manwell LB, Linzer M, Brown RL, Schwartz MD. Burden of difficult encounters in primary care: data from the minimizing error, maximizing outcomes study. Arch Intern Med. 2009;169(4):410-14.

4. Zeiss AM, Karlin BE. Integrating mental health and primary care services in the Department of Veterans Affairs health care system. J Clin Psychol Med Settings. 2008;15(1):73-8.

5. Kwan B, Nease D Jr. The state of the evidence for integrated behavioral health in primary care. In: Talen MR, Valeras AB, eds. Integrated Behavioral Health in Primary Care. New York: Springer; 2013:65-98.

6. Miller BF, VanderWielen LM, Melek S, Kathol R, Davis M, Gordon P. Payment reform in the patient centered medical home: sustaining integrated care for behavioral health. Am Psychol. Under review. 